\title{
Magnetic field dependance of the plasma properties in a negative hydrogen ion source for fusion
}

\author{
L. Schiesko, P. McNeely, P. Franzen, U. Fantz and the NNBI \\ Team \\ Max-Planck-Institut für Plasmaphysik, EURATOM Association, Boltzmannstrasse 2, \\ D-85748 Garching, Germany \\ E-mail: loic.schiesko@ipp.mpg.de
}

\begin{abstract}
Axially resolved measurements of plasma parameters were performed by two Langmuir probes moving in parallel from the exit of the driver (where the plasma is generated) up to the extraction region neighbourhood of the IPP RF negative hydrogen ion source prototype. At the driver exit, the plasma parameters show an unexpected inhomogeneity in the presence of the magnetic field: a cold and dense plasma is flowing out of the top part of the driver while a hot and low density plasma flows from the bottom part. A local relation between the top and bottom parameters is derived from the conservation of the energy flux.
\end{abstract}

\section{Introduction}

The key component of the ITER NBI system is the negative hydrogen ion source. For heating and current drive, ITER requires $40 \mathrm{~A}^{-}$of $\mathrm{D}^{-}$accelerated to $1 \mathrm{MeV}$. In 2007 the IPP RF prototype source was chosen as the reference design for the plasma generation in the ITER NBI system $[1,2,3,4]$. The extraction of negative ions inevitably leads to the co-extraction of electrons. In order to reduce electron power loading on the 
Magnetic field dependance of the plasma properties in a negative hydrogen ion source for fusion 2 accelerator the ratio of extracted electron to ion must be below unity. A magnetic filter field, generated by permanent magnets located near the diagnostic flange, cools down the electron temperature, and thereby diminishes the destruction of the negative ions by electron collisions. Due to simultaneously decreasing the density and temperature it also reduces the number of co-extracted electrons. The magnetic filter field position, strength and topology are, however, not optimized. Compared to previous studies [1], the magnets are now installed in a frame capable of being moved from the driver exit (close to the plasma generation zone) to the extraction region. Details on this frame are given in section 2 [5]. This setup provides a greater experimental flexibility for investigating the effect of the magnetic field location on the plasma and the negative ion beam. A recent study shows the influence of the position and the strength of the magnetic field on the amount of extracted negative ions and co-extracted electrons [5]. From this study, two correlations were found: for the negative ion extraction, the important parameter is the magnetic field strength in front of the plasma grid $(\mathrm{PG})$, while for the reduction of the co-extracted electron amount, the $\int B d z$ from the driver to the PG is the key parameter.

One of the goals of this paper is to determine the influence of the magnetic field position on the plasma parameters in the expansion region of the source. Also a comparison will be made between the results obtained with and without magnetic filter field. Finally, a local relation between the plasma parameters obtained by each probe will be derived by using the conservation of the mean energy flux. 


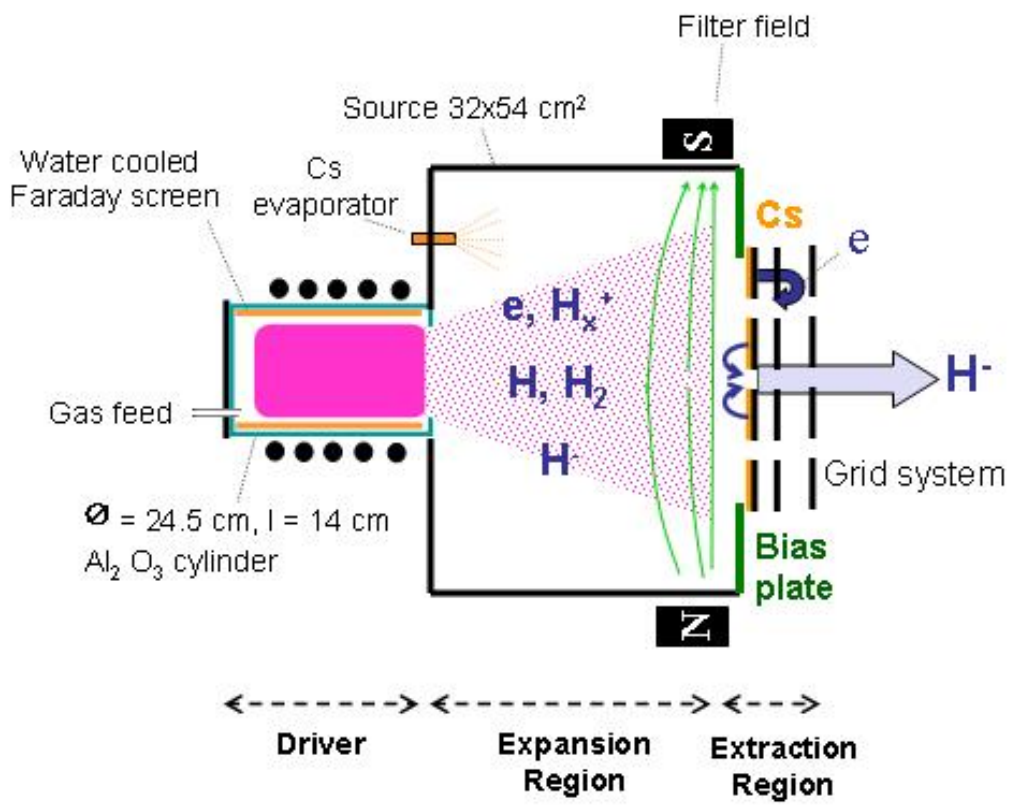

Figure 1. Top view of the IPP prototype source, the magnets can be moved along the expansion region

\section{Experimental setup}

\subsection{The IPP negative hydrogen ion source prototype}

The experiments were conducted at the BATMAN testbed [1]. A schematic representation of the source can be seen on figure 1. The source is set at a high negative potential $(-20 \mathrm{kV})$. It is an inductively coupled source with a maximum RF power of $100 \mathrm{~kW}$ at

$1 \mathrm{MHz}$. The plasma is generated in the driver via a water cooled coil antenna, and then diffuses into the expansion chamber. An internal Faraday shield is installed in the driver in order to protect the alumina cylinder from the thermal effects and plasma erosion. In order to extract a large amount of negative ions with a low co-extracted electron fraction, the evaporation of Cs is mandatory. The negative ions are generated by the 

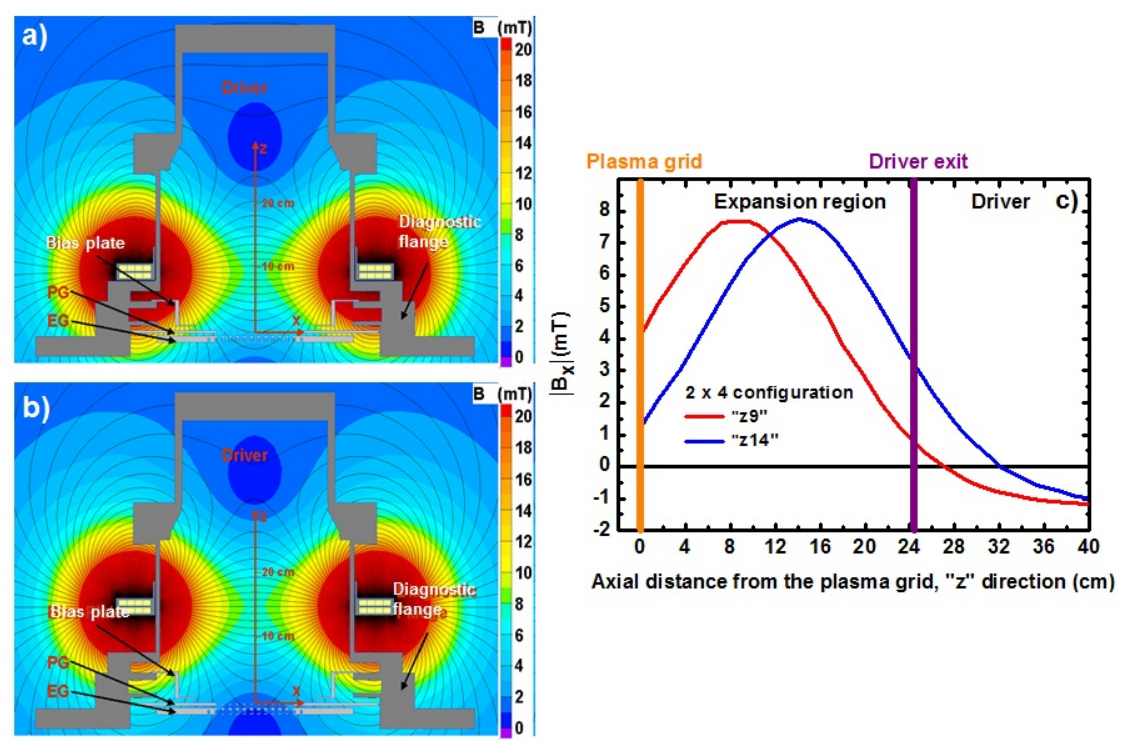

Figure 2. a) and b) represent the magnetic field map of the horizontal cut of the source for the "z9" and "z14" case respectively. c) is the horizontal magnetic field strength variation in the " $z$ " direction. The zero of the referential was taken at the center of the PG. The $(\mathrm{x}, \mathrm{z})$ plane corresponds to the horizontal plane.

conversion of neutrals and positive ions on the plasma grid. It has been long known that the addition of Cs in a source reduces the workfunction of the surfaces, which increases the negative ion yield $[11,12,13]$. In the present study, the source was already cesiated (details in [4]), and the Cs oven was set to a low temperature $\left(140-160^{\circ} \mathrm{C}\right)$ in order to maintain a high and stable source performance.

The extraction system is composed of three grids (see [1] for full details). Briefly, the first grid is the PG, where most of the extracted negative ions are generated and the second grid is the extraction grid (EG) which holds permanent magnets to deflect the co-extracted electrons out of the beam (these magnets are not the ones generating the magnetic filter field). The last grid is the grounded grid. Typically, at BATMAN and for technical reasons, the voltages are $-20,-11$ and $0 \mathrm{kV}$ for the plasma, extraction and grounded grid.

In this prototype source, the expansion region and the extraction region are separated by a magnetic filter field generated by permanent magnets. In contrast to previous 
Magnetic field dependance of the plasma properties in a negative hydrogen ion source for fusion5 studies, a new movable frame for the magnets allows more flexibility [5]. The magnets can be moved along the expansion chamber (see figure $2 \mathrm{a}$ and $\mathrm{b}$ for two examples of magnets locations), from the PG neighbourhood up to the driver exit. The results obtained when the magnets are located as close as possible to the driver exit will not be presented due to a poor source performance (low extracted negative ions and high electron to negative ion ratio) and a low signal to noise ratio with the Langmuir probes. It is not possible at BATMAN to sustain the plasma at low pressures (e.g. $0.3 \mathrm{~Pa}$ ) when the magnetic field is shifted towards the driver. Two reasons may explain this difficulty. First, the magnetic field penetrates inside the driver as can be seen on figures 2, second, high neutral depletion was measured in the driver at BATMAN [6] because of the high injected RF power. This is why this study was conducted at $0.6 \mathrm{~Pa}$, where no issue was encountered. This issue was not encountered at RADI $[7,8]$, which could be operated at $0.2 \mathrm{~Pa}$ with a strong magnetic field inside the drivers. This is probably due to the larger size of RADI, which is equipped of four drivers and for which the gas flow is much higher than for BATMAN. The size of the ITER NBI system being expected to be two times bigger than for RADI, the plasma should be sustained at low pressure without any issue. Figures $2 \mathrm{a}$ and $2 \mathrm{~b}$ present the $2 \mathrm{D}$ map of the magnetic field configuration obtained by eight magnets on each side (labelled as " $2 \times 4$ "). The two magnets positions presented on figure $2 \mathrm{a}$ and $2 \mathrm{~b}$ will be labelled as the " $\mathrm{z} 9$ " and "z14" for the maximum of the field being achieved at $9 \mathrm{~cm}$ and $14 \mathrm{~cm}$ from the PG respectively (see figure 2c). For all the cases and locations, the $(y, z)$ is a symmetry plane of the magnetic field lines. Figure 2c presents for the two cases, the horizontal magnetic field strength along the $\mathrm{z}$ axis. 
Magnetic field dependance of the plasma properties in a negative hydrogen ion source for fusion6

\subsection{Diagnostic}

2.2.1. Langmuir probes For this study, two RF compensated movable cylindrical Langmuir probes were used. The analysis system is the PlasmaMeter developed at the School of Physics and Technology of the K.N. Karazin Kharkov National University, Ukraine. The Langmuir probe analysis system and RF compensation was previously described in great detail [10]. The probe tips are made of tungsten with a radius of $25 \mu \mathrm{m}$ and a length of $0.3 \mathrm{~cm}$. Figure 3 shows the exact probe location. The probes are located at the $\mathrm{x}=5.0$ (in $\mathrm{cm}$ ) position and move in the $\mathrm{z}$ direction, on the $(5.0, \pm 10.0, \mathrm{z})$ plane. As can be seen, the probes have an allowed movement range of $22.0 \mathrm{~cm}$ from the driver exit, up to the neighbourhood of the PG. The direction of the magnetic field lines is indicated on figure 3. The $(\mathrm{y}, \mathrm{z})$ plane corresponds to the vertical plane while the $(\mathrm{x}, \mathrm{z})$ plane is the horizontal plane. For convenience, in the following discussion, the probes will be labelled as "top" for the probe in the upper part of the figure $3(5.0,10.0, \mathrm{z})$ and "bottom" for the probe in the lower part $(5.0,-10.0, \mathrm{z})$.

2.2.2. Data evaluation and discussion of errors The plasma potential was evaluated at the zero-crossing of the second derivative of the I-V curve of the probe. For all the measurements, the zero-crossing point of the second derivative was well defined and the difference between the maximum and the minimum of the second derivative was lower than $\mathrm{kT}_{e} / \mathrm{e}$. Typically, $\mathrm{kT}_{e} / \mathrm{e}$ was in the order of $1 \mathrm{~V}$.

The electron temperature was evaluated by a linear approximation of the logarithm of the second derivative. For high electron temperature (driver exit) the error is small. When moving towards the PG, the plasma density and electron temperature decrease. Due to a lower signal to noise ratio the error is larger in this region, $\Delta \mathrm{T}_{e}=0.5 \mathrm{eV}$ (as indicated on figures $4 \mathrm{~b}$ and $5 \mathrm{~b})$. 

The ion saturation current was taken at a potential of $\mathrm{V}_{p}-10 \mathrm{kT}_{e}$ for each measurement. At a such potential, the contribution of the high energy electrons to the current is negligible. The systematic error in the probe current measurement is roughly $20 \mu \mathrm{A}$. The accuracy in the ion density evaluation is limited by the knowledge of the exact ion mass, a direct measurement of the proportion of each ion species $\left(\mathrm{H}^{+}, \mathrm{H}_{2}^{+}\right.$and $\left.\mathrm{H}_{3}^{+}\right)$ being not possible. In principle, the ion mass vary between 1 and 3. An estimation of the effective mass can be done provided the measurements of the species distribution for positive $\mathrm{H}$ ion beams extracted from other RF sources. Pressure and RF power scan were performed at a source [15] (functionally identical to the driver of the ion source used in this study) operated for the decomissioned W7-AS stellerator. Results found a proportion of $40 \% \mathrm{H}^{+}, 40 \% \mathrm{H}_{2}^{+}$and $20 \% \mathrm{H}_{3}^{+}$resulting in 1.8 being the most probable effective mass. The contribution of $\mathrm{Cs}^{+}$ions to the probe current can be neglected as their density is small compared to the hydrogen ions species $\left(1 \%\right.$ of $\left.n_{e}[16]\right)$ and due to their smaller velocity resulting from their higher mass. The positive ion density was evaluated by the Kiel approximation for BRL (Bernstein-Rabinowitz-Laframboise) [17], and both the ABR (Allen-Boyd-Reynolds) approximation [18] and the Chen formula for OML (Orbital Motion Limited) [19]. The latter allows for the determination of the the ion density without having to know the electron temperature. A good agreement between the three evaluation techniques was found. The best agreement between the positive ion density and electron density was given by the Chen formula.

The presence of a magnetic field can be an issue in the evaluation of the electron density. It is known that a magnetic field can substantially reduce the electron current collected by a Langmuir probe [20]. However, an estimation for $\mathrm{T}_{e}=1 \mathrm{eV}, \mathrm{B}=8 \mathrm{mT}$, which is the typical magnetic field strength, shows that the electron gyroradius $(300 \mu \mathrm{m})$ greatly exceeds the probe tip radius $(25 \mu \mathrm{m})$. The magnetic tube surface area for a radius equal 


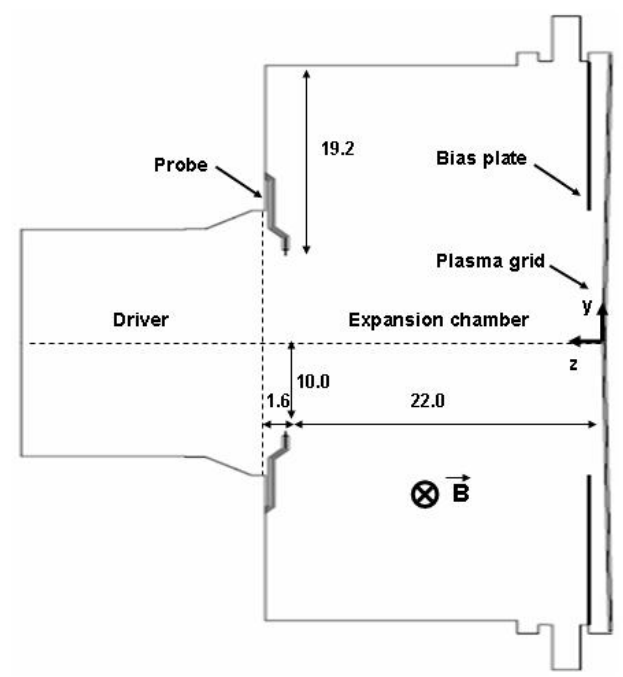

Figure 3. Location of the Langmuir probes. The distances are in $\mathrm{cm}$. The magnetic field direction is indicated. The (y,z) plane corresponds to the vertical plane. Dimensions in $\mathrm{cm}$.

to the electron gyroradius greatly exceeds the probe surface area. As a consequence, the influence of the magnetic field on the electron saturation current of the probes is likely negligible. This simple estimation is in good agreement with the Langmuir probe measurements performed at RADI [21] where the two recorded probe traces, one with the probe tip parallel and the other one with the probe tip perpendicular to the magnetic field lines could be superimposed without any significant difference.

Typically, the probe tip temperature increases beyond $1000 \mathrm{~K}$ during measurements, therefore probe contamination by $\mathrm{Cs}$ is not considered probable. The measurements being made at a large distance of the the PG (see figure 4), the negative ions generated on the PG surface should not affect the Langmuir probe measurements. Moreover, the electronegativity of the bulk plasma being low, the negative ions created in the volume should not drastically change the shape of the Langmuir probe traces. 
Magnetic field dependance of the plasma properties in a negative hydrogen ion source for fusion 9

\section{Results and discussion}

\subsection{Influence of the magnetic field position on the plasma parameters}

The experiments were conducted at a pressure of $0.6 \mathrm{~Pa}$ in hydrogen, and an injected RF power of $40 \mathrm{~kW}$. The extraction voltage was set to $9 \mathrm{kV}$ and the magnetic field direction is as indicated in figure 3. The figures 4,5 and 6 present the variation of the positive ion density, electron temperature and plasma potential obtained by the two movable Langmuir probes for the "z9" and "z14" magnetic field positions, and for the case without magnetic field respectively. The magnetic field orientation was the same for all the measurements (see figs. 4, 5). On figure $5 \mathrm{a}$, at the positions $\mathrm{z}=2 \mathrm{~cm}$ and $\mathrm{z}=4 \mathrm{~cm}$, the signal to noise ratio was sufficient in the ion branch to determine the positive ion density via the Chen formula. It was however not possible to derive the electron temperature or the plasma potential in the electron branch for these positions (see figures $5 \mathrm{~b}$ and $\mathrm{c}$ ).

On figure $4 \mathrm{a}$ and $5 \mathrm{a}$ one can see that the plasma density measured right at the exit of the driver is already very inhomogenous: the ion density on the top probe greatly exceeds the one mesured by the bottom probe. The effect is stronger in the "z14" configuration. There is a strong difference in the density profiles along the $\mathrm{z}$ axis between the two configurations. Although a continuous decrease of the ion density is observed by the top probe for both magnet positions, the bottom probe shows a completely different behavior. The data from the bottom probe (see fig. 4a and 5a), gives a maximum located at the position where the magnetic field is the strongest. Taking into consideration that losses onto the walls should occur everywhere in the expansion region, it is odd that a maximum exist at a location so far from the driver. Measurements in arc sources find inhomogeneous plasmas as well [22]. The inhomogeneity for the arc source is due to a $\mathbf{B} \times \nabla \mathbf{B}$ drift of the fast electrons. This is different than what is observed in this 


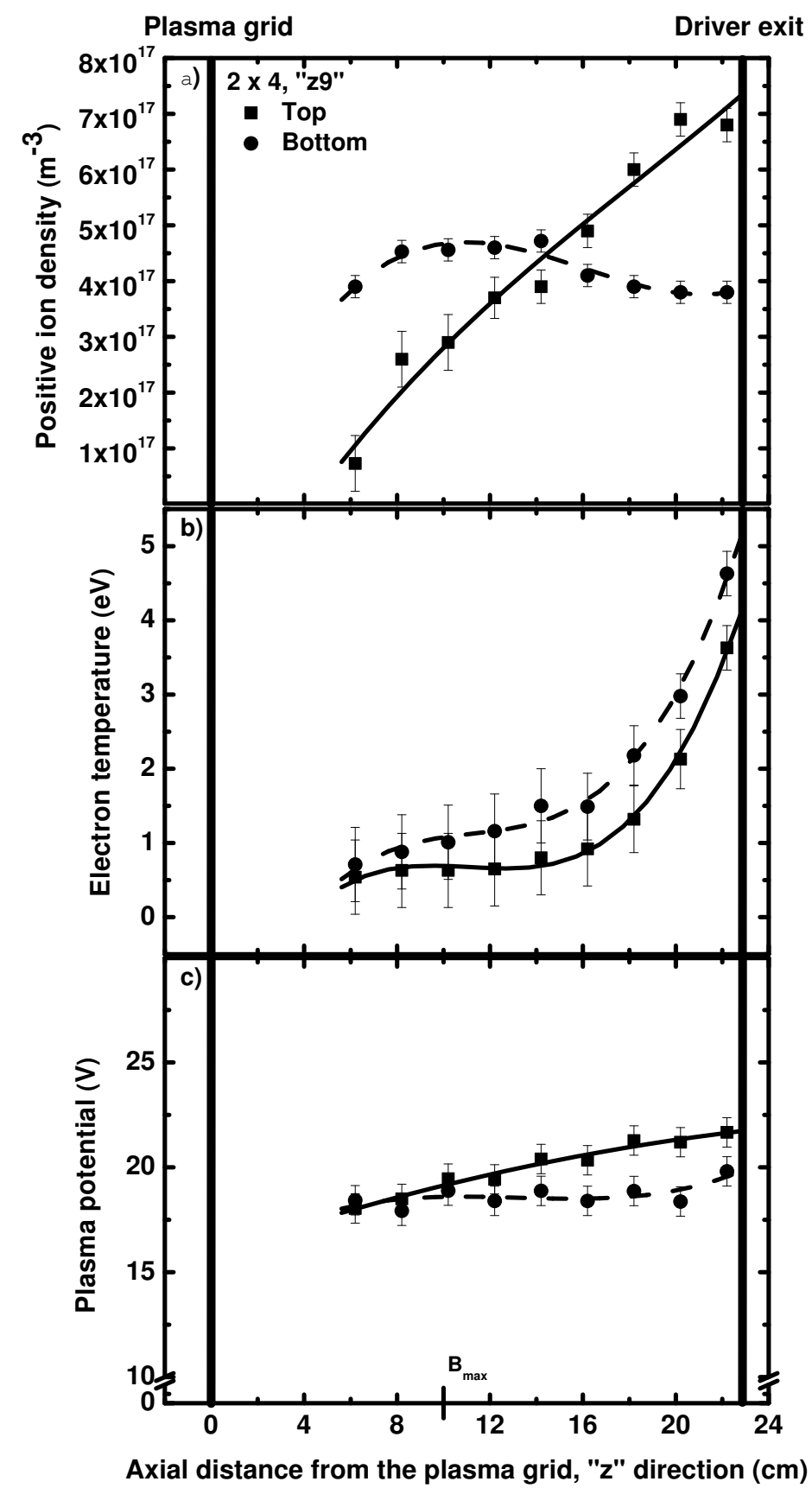

Figure 4. a) and b) and c) represent the variations of the positive ion density, electron temperatures and plasma potential for the two Langmuir probes, moving along the expansion chamber and for the " $z 9 "$ magnetic field configuration. Straight and dashed lines refer to the top and bottom probe respectively. The position of the maximum magnetic field strength is indicated. 


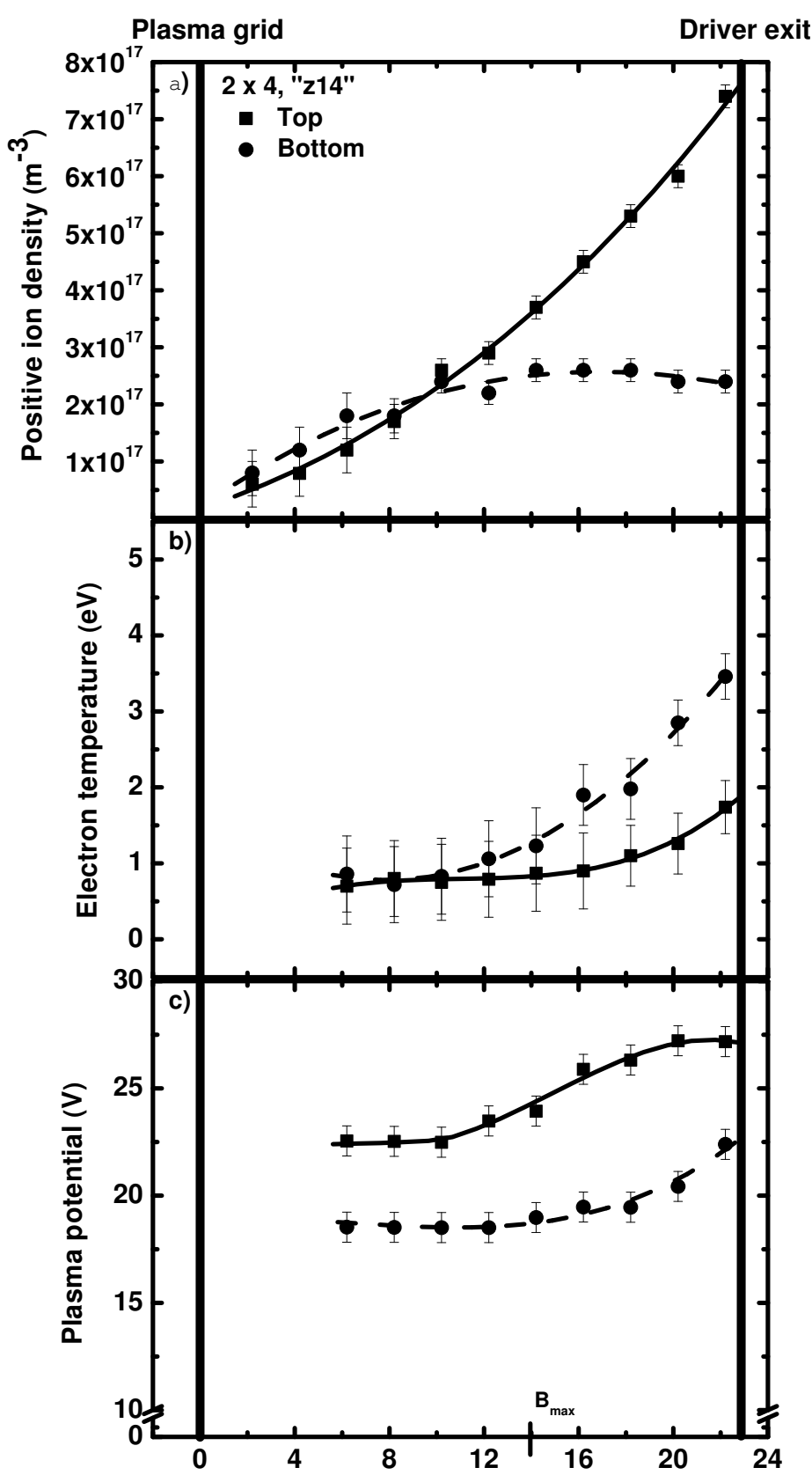

Axial distance from the plasma grid, " $z$ " direction $(\mathrm{cm})$

Figure 5. a) and b) and c) represent the variations of the positive ion density, electron temperatures and plasma potential for the two Langmuir probes, moving along the expansion chamber and for the "z14" magnetic field configuration. Straight and dashed lines refer to the top and bottom probe respectively. For the positions $\mathrm{z}=2$ and 4 , only the positive ion density could be derived via the Chen formula due to a poor signal to noise ratio in the electron branch. The position of the maximum magnetic field strength is indicated. 


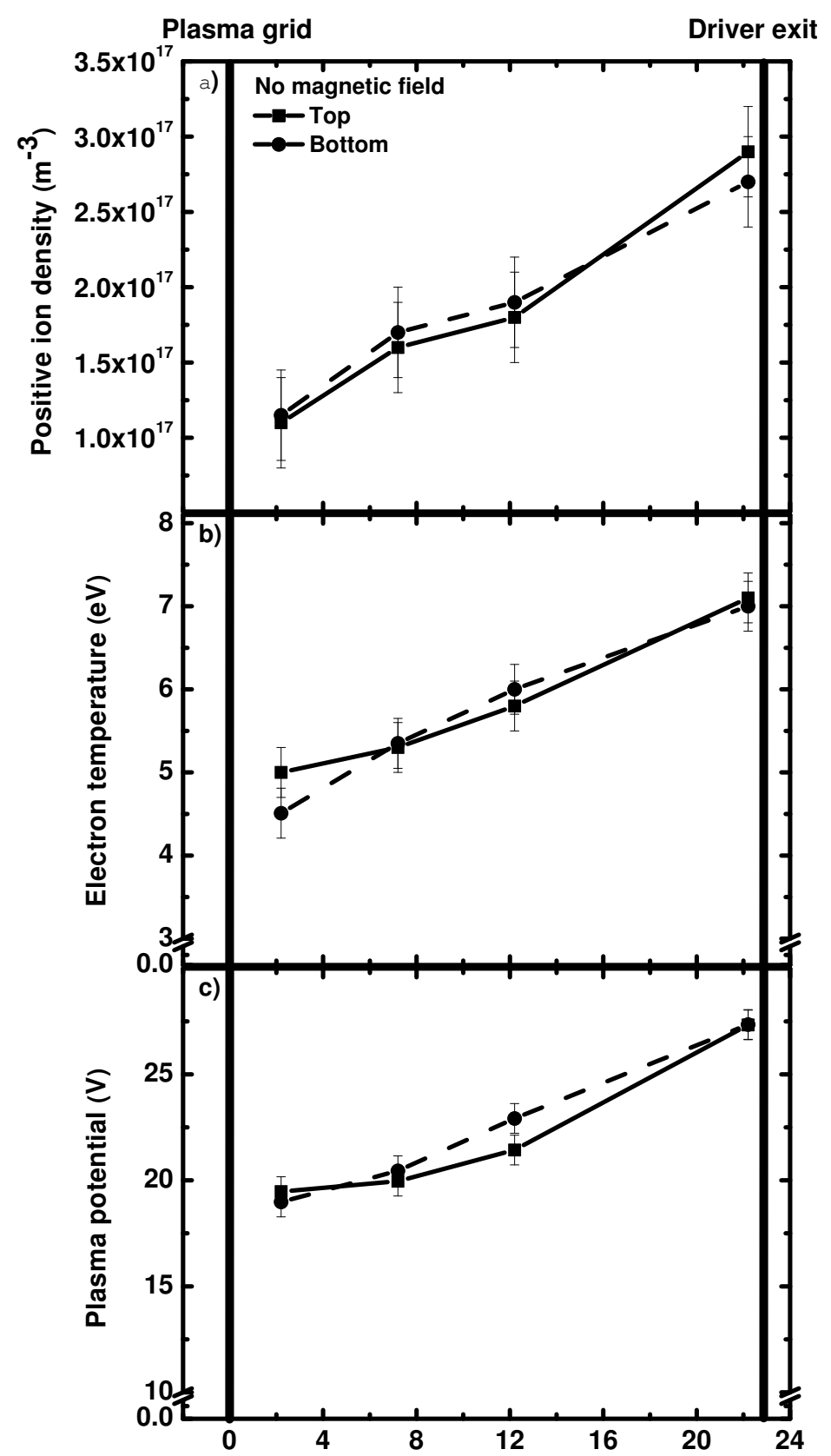

Axial distance from the plasma grid, " $\mathrm{z}$ " direction (cm)

Figure 6. a) and b) and c) represent the variations of the positive ion density, electron temperatures and plasma potential for the two Langmuir probes, moving along the expansion chamber and without the presence of the magnetic field. Straight and dashed lines refer to the top and bottom probe respectively. 
Magnetic field dependance of the plasma properties in a negative hydrogen ion source for fusion 13

work as the inhomogeneous plasma is due to an inhomogeneous fast electron density in [22]. In arc sources, the plasma is created primilarily via collisions with the fast primary electrons. In this work the the plasma being observed is the secondary plasma from the driver. As the plasma is generated in the driver via the induced loop current there is no obvious reason for an inhomogeneity this close to the driver exit. 2D PIC-MCC codes $[23,24]$ and 2D Fluid models [25] also deal with magnetized hydrogen plasmas. In the Kollev paper [25], a maximum in density is observed when the magnetic field reaches its maximum intensity. This is due to the decrease of the electron temperature leading to an accumulation of electrons. It is possible that the maximum observed on bottom probe could be due to the same effect, however; as the electron temperature decreases for both probes, a maximum should also be observed on the top probe. Moreover, the magnetic field configuration chosen in [25] is completely different from the one at BATMAN. The fact that the plasma flowing out of the driver is already inhomogeneous will be discussed later.

When no magnetic field exists, the plasma is homogeneous and the ion density decreases towards the PG as can be seen in figure 6a. It can then be concluded that the behavior of the plasma within the expansion chamber is due to the presence of the magnetic field.

On figure $4 \mathrm{~b}$ and $5 \mathrm{~b}$, it is observed that for the same magnetic field configuration, the top and bottom probes measure a very different electron temperature at the driver exit. This difference is attenuated proceeding away from the driver due to the influence of the magnetic field cooling down the electrons. Like for the density, in the "z14" configuration the difference between top and bottom is more pronounced. In the "z9" configuration both top and bottom temperatures are lower near the driver exit than in the "z14" configuration. This suggests that the mechanism leading to the inhomogenity 
Magnetic field dependance of the plasma properties in a negative hydrogen ion source for fusion 14 at the driver exit is proportional to the $\int B d z$ encountered by the charged particles. The field penetrates deeper into the driver for the "z14" than for the "z9" case (see figure 2c). The deeper penetration of the magnetic field into the driver for the "z14" case also explains why the electron temperature in the neighbourhood of the driver exit is systematically lower than for the "z9" configuration. Again, no significant difference between top and bottom temperatures is seen wihout magnetic field in figure $6 \mathrm{~b}$. The measured electron temperature without magnetic field is also greater than what it is measured with a magnetic field, especially near the PG; as expected.

The plasma potential variations due to the magnetic field can be seen in figure $4 \mathrm{c}$ and 5c. A different plasma potential is measured at the top and bottom parts of the expansion chamber. In the "z9" configuration the plasma potentials are essentially identical by the middle of the expansion chamber. In the "z14" configuration there is a strong potential difference that remains all along the expansion region. This stronger difference between top and bottom that occurs for the "z14" configuration demonstrates that this phenomenom is related to the encountered $\int B d z$, as no significant difference is seen between the probes when no field is present, and the effect is reduced for the " $z 9$ " configuration.

A simple determination of the $\mathbf{E} \times \mathbf{B}$ direction taking the magnetic field vector from figure 3, and the electric field direction determined by the variation of the plasma potential within the expansion region shows that this particular drift points upwards. The determination of the $\nabla \mathbf{P} \times \mathbf{B}$ points toward bottom. This suggests that the observed behaviors are complex and that a combination of several cross-B drifts could be happening. Thus a solid 3D modelling of the source is required to determine the underlying mechanisms. 
Magnetic field dependance of the plasma properties in a negative hydrogen ion source for fusion 15

Measurements performed with the reversed magnetic field direction (not presented in this paper) show the same behavior but with the properties of the plasma in the top and bottom being reversed, confirming that cross-B drifts are likely responsible of the observed behaviours.

From these previous measurements it is clear there is a cold and dense plasma flowing out of the top part of the driver, while a hot and low density plasma comes out of the bottom part of the driver. It is not a trivial observation that the plasma flowing out of the driver is strongly inhomogenous when a magnetic field is present. Indeed, in the driver, the power is inductively coupled to the gas by a 6 turn coil, and it is unlikely that the power density of the inducted current is inhomogeneous. This is confirmed by the observation that without a magnetic field, no inhomogeneity is seen at the driver exit. With respect to the plasma potential profiles of figure $4 \mathrm{c}$ and $5 \mathrm{c}$, it is odd to observe that the electron temperature in the bottom part (lower plasma potential) is higher than in the top part (higher plasma potential). The plasma potential in the driver [10] has been measured to be higher than in the expansion chamber. Assuming an homogeneous plasma potential in the driver, then for the potential measured by the bottom probe being lower than for the upper probe, it would be expected that the temperature should be lower at the bottom probe, contrary to what is measured. Two hypotheses may explain the observed behaviours. First, it is possible that the magnetic field penetrating inside the driver induces an inhomogenity in it. The plasma potential, density and temperature could be different in the top and bottom part of the driver, leading to a higher density flowing out of the top part of it. The continuous decrease of the top density and the maximum oberved by the bottom probe in the expansion region could be due to the plasma twisting downwards because of a combination of cross $\mathrm{B}$ 
Magnetic field dependance of the plasma properties in a negative hydrogen ion source for fusion 16 drifts. Measurements are planned to be performed in the driver by a rotatable L-shaped Langmuir probe to determine whether or not the plasma parameters are homogeneous. Another possibility is that the plasma is flowing out of the driver with an angle with the $\mathrm{z}$ direction when the magnetic field is present. This could also explain the observed results.

\subsection{A local relation between the densities and temperatures evaluated at the top and bottom parts of the expansion region}

In this section will be derived a relation between the electron temperature, $\mathrm{T}_{e}$, and the electron density, $\mathrm{n}_{e}$, between the top and bottom part of the expansion chamber.

On the plane of symmetry $\mathrm{x}=0$, where the probes lie, the electron energy balance is

$$
\operatorname{div} \mathbf{Q}_{\mathbf{e}}=P_{h}-P_{l}
$$

which can be rewritten as

$$
\frac{\partial Q_{y}}{\partial y}+\frac{\partial Q_{z}}{\partial z}=P_{h}-P_{l}
$$

with $\mathrm{P}_{h}$ is a heating source term, $\mathrm{P}_{l}$ is the power loss due to inelastic collisions and losses to the walls $\left(\partial \mathrm{Q}_{x} / \partial \mathrm{x}\right)$, while $\mathrm{Q}_{y}$ and $\mathrm{Q}_{z}$ are the $\mathrm{y}$ and the $\mathrm{z}$ components of the energy flux respectively. In the expansion region, far from the source of particles, $\mathrm{P}_{h}=0$. The drifts induced by the presence of the magnetic field create an asymmetry between top and bottom in the y direction through the term $Q_{y}$. The main energy flow being the $\mathrm{z}$ direction, the $\mathrm{Q}_{y}$ term can then be neglected. The equation is integrated along the $\mathrm{z}$ axis at the position of the probes $\mathrm{y}=\mathrm{y}_{t}$ and $\mathrm{y}=\mathrm{y}_{b}\left(=-\mathrm{y}_{t}\right)$

$$
\begin{aligned}
& Q_{z, t}(z)=Q_{z, t}\left(z_{0}\right)-\int P_{l, t} d z \\
& Q_{z, b}(z)=Q_{z, b}\left(z_{0}\right)-\int P_{l, b} d z
\end{aligned}
$$

the suffixes $t$ and $b$ representing top and bottom probes and $z_{0}$ some position in the driver. Assuming that the energy in the driver is coupled homogeneously to the gas, 

then, $\mathrm{Q}_{z, t}\left(z_{0}\right)=\mathrm{Q}_{z, b}\left(z_{0}\right)$. If it is assumed that $\mathrm{P}_{l}$ is a function of $\int \mathrm{Bdz}$ only, which is symmetrical with respect to the $(\mathrm{x}, \mathrm{z})$ plane, we have $\mathrm{P}_{l, t}=\mathrm{P}_{l, b}$. Thus

$$
Q_{z, t}(z)=Q_{z, b}(z)
$$

Under the assumption of a Maxwellian energy distribution function of the species, the energy flux can be expanded into its conductive and convective part and (5) becomes

$$
q_{z, t}(z)+\frac{3}{2} k T_{e, t}(z) \Gamma_{z, t}(z)=q_{z, b}(z)+\frac{3}{2} k T_{e, b}(z) \Gamma_{z, t}(z)
$$

with $\Gamma_{z}(\mathrm{z})$ the particle flux, $\mathrm{q}$ being the conductive term and $(3 / 2) \mathrm{kT}(\mathrm{z}) \Gamma_{z}(\mathrm{z})$ the convective part. The particle flux $\Gamma_{z}(\mathrm{z})$ is balanced by particle flux to the wall $\Gamma_{x}(\mathrm{z})$ along the magnetic field lines so that

$$
\Gamma_{z}(z) \propto \Gamma_{x}(z)=\frac{1}{4} v_{t h, e} n_{e}
$$

Assuming that $\mathrm{q}_{z, t}(\mathrm{z})=\mathrm{q}_{z, b}(\mathrm{z})$ it follows from $(6)$

$$
n_{e, t}(z) k T_{e, t}(z) v_{e, t}(z)=n_{e, b}(z) k T_{e, b}(z) v_{e, b}(z)
$$

Provided that $\mathrm{v} \propto \mathrm{T}_{e}^{1 / 2}$ it follows

$$
n_{e, t}(z) T_{e, t}^{3 / 2}(z) \simeq n_{e, b}(z) T_{e, b}^{3 / 2}(z)
$$

When no magnetic field is present and due to source symmetry, the relation (9) is shown to be valid by figure 7a. The decrease of the mean energy flux towards PG is due to inelastic collisions between electrons and neutrals and the loss of electrons to the walls. Figure 7a confirms that the energy is coupled homogeneously to the gas in the driver when no magnetic field is present. In figure $7 \mathrm{~b}$ and $7 \mathrm{c}$ one can see that the relation (9) is still valid between the top and bottom for each z position and for both the "z9" and "z14" cases. The small discrepancy observed on figure $7 \mathrm{~b}$ may come from the fact that relation (9) is obtained ignoring cross field drifts $\left(\mathrm{Q}_{y}\right.$ and $\left.\Gamma_{y}\right)$. The validity of the relation (9) confirms the assumption made previously that $\mathrm{q}_{z, t}(\mathrm{z})=\mathrm{q}_{z, b}(\mathrm{z})$. In order to 


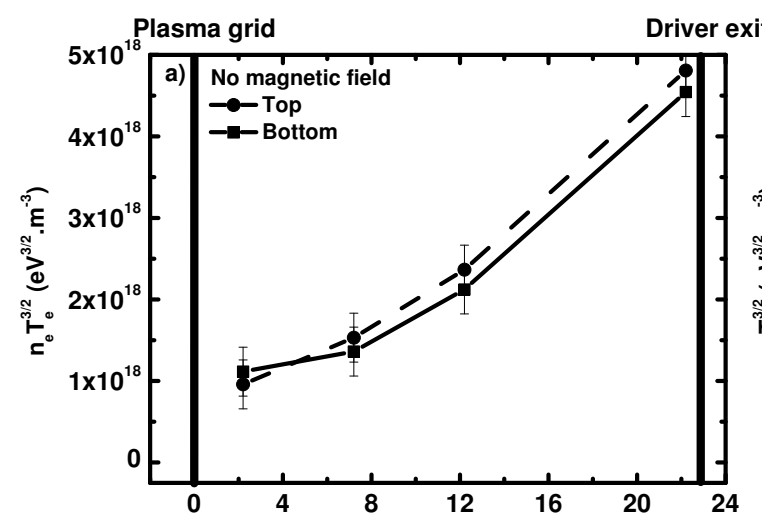

Axial distance from the plasma grid, " $\mathrm{z}$ " direction (cm)

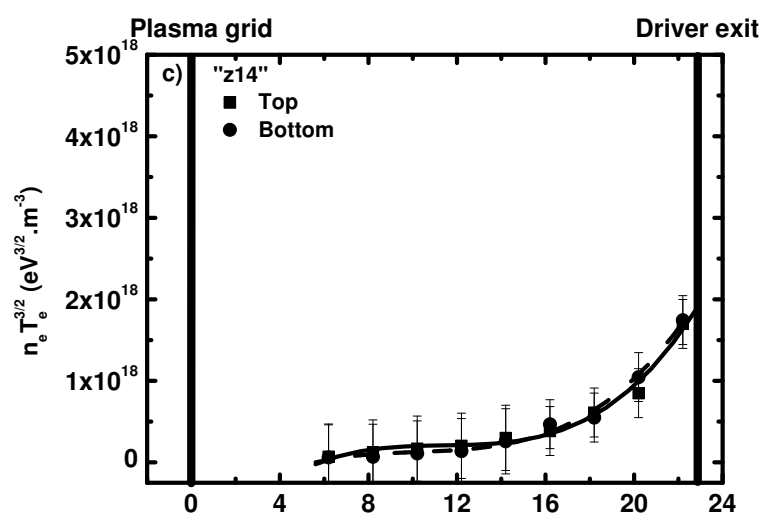

Axial distance from the plasma grid, " $\mathrm{z}$ " direction (cm)

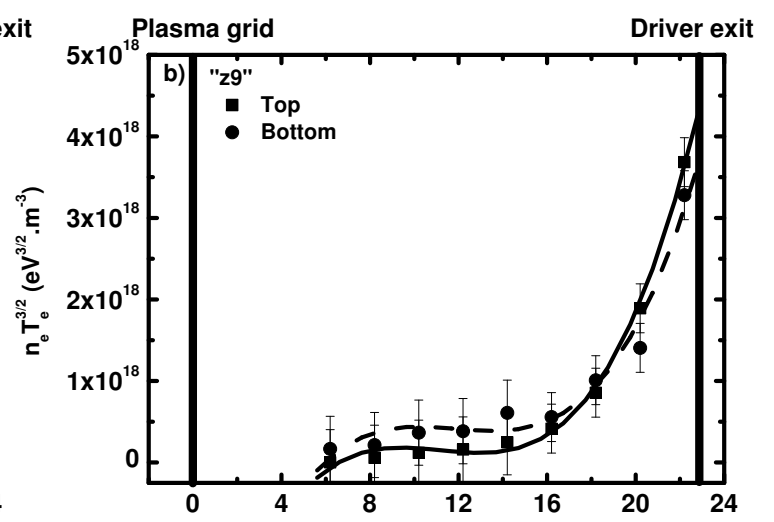

Axial distance from the plasma grid, " $\mathrm{z}$ " direction (cm) Plasma grid

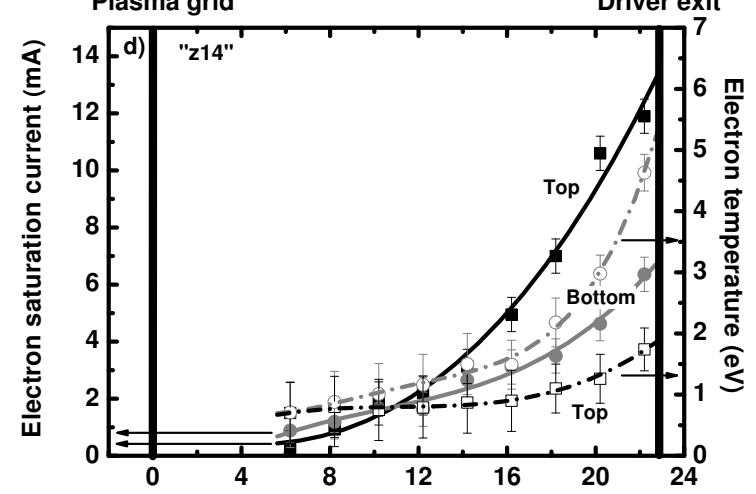

Axial distance from the plasma grid, " $\mathrm{z}$ " direction $(\mathrm{cm})$

Figure 7. Plot of variious quantities versus the axial distance from the plasma grid: a) $\mathrm{n}_{e} \mathrm{~T}_{e}^{3 / 2}$ without magnetic field, b) $\mathrm{n}_{e} \mathrm{~T}_{e}^{3 / 2}$ in the " $\mathrm{z} 9$ " configuration, c) $\mathrm{n}_{e} \mathrm{~T}_{e}^{3 / 2}$ in the "z14" configuration and d) the electron saturation current and associated electron temperature for the "z14" case. Straight and dashed lines correspond to top and bottom probes respectively on figures a), b) and c). On figure d) straight black and grey lines correspond to the top and bottom electron saturation current respectively, while the dashed black and grey lines are related to the top and bottom electron temperature respectively.

understand this behavior, the electron saturation current with the associated electron temperature is plotted on figure $7 \mathrm{~d}$ for the "z14" configuration. From this it can be seen that at any location, especially for the positions close to the driver exit, the lack of electron current in the bottom (full circles, lower density) is exactly compensated by a higher temperature (open circle). On the contrary, the higher electron current measured in the top part (full squares, high electron density) is associated with a lower electron temperature (open squares). This is also true for the "z9" case (not shown 
Magnetic field dependance of the plasma properties in a negative hydrogen ion source for fusion 19 here). Figures $7 \mathrm{~b}$ and $7 \mathrm{c}$ also show the way the energy is lost by a plasma flowing into a transverse field. When comparing the "z9" to the "z14" case it can be seen that as less field is present in the driver there is more energy is flowing out of the driver. The conservation of the mean energy flux between the top and bottom of the plasma is an indication that the properties of the plasma are determined by the magnetic field configuration and that the energy is still homogeneously coupled to the gas in the driver. The strong relation between the magnetic field and the plasma parameters suggests that the relation (9) is local, that is only valid for particles that have experienced the same $\int B d z$. If we consider the same $(\mathrm{x}, \mathrm{z})$ plane, then the magnetic field lines being curved, the charged particles encounter a field strength that depends of their z position on the same $(x, z)$ plane, as can be seen on figures $2 a$ and $2 b$. The magnetic field lines being symmetric with respect to the $(\mathrm{x}, \mathrm{z})$ plane, it can then be concluded that the $(\mathrm{x}, \mathrm{z})$ plane is a symmetry plane for the relation (9). These considerations and relation (9) also hold when the electron density is replaced by the ion density. This is due to the fact that the ions enter the sheath with the Bohm velocity, which is dependent on the electron temperature.

\section{Conclusion}

The goal of this paper was to determine the influence of the magnetic field position on the plasma parameters derived from Langmuir probe measurements in the expansion chamber of the IPP RF negative ion source. The use of two movable Langmuir probes showed a strong and quite unexpected plasma inhomogeneity between the top and bottom part of the expansion chamber. The strongest inhomogeneity between top and bottom plasma parameters was observed to be at the driver exit. It is a reasonable assumption that the interaction of several cross-B drift plays a key role in producing 
Magnetic field dependance of the plasma properties in a negative hydrogen ion source for fusion 20

this inhomogeneity. It is possible to rule out a non-homogeneous heating in the driver as a cause of these measurements by the observation that under all experimental conditions there is a conservation of the mean energy flux. This conservation allowed for the derivation of a simple local relation between the top and bottom temperatures and densities. When the magnetic field is present, the observation of the plasma inhomogeneity right at the driver exit suggests that despite the energy of the driver being coupled into the plasma uniformly, the potentials, temperatures and densities could show a significant difference between the top and bottom part of it. In the near future measurements will be performed from backplate of the driver up to the PG in order to better qualify the plasma properties in the driver and in the, as yet unexplored, transition driver / expansion region.

\section{Acknowledgements}

The authors would like to thank one of the referees for making the derivation of eq.

(9) more rigorous. The work was supported by a grant from Fusion for Energy (F4E GRT-313) supervised by Antonio Masiello. The opinions expressed herein are those of the authors only and do not represent the Fusion for Energy's official position. 
Magnetic field dependance of the plasma properties in a negative hydrogen ion source for fusion 21

[1] E. Speth et al. Nucl. Fus., 46, S220, 2006.

[2] U. Fantz et al. Nucl. Fus., 49, 125007, 2009.

[3] McNeely P. and Schiesko L. Rev. Sci. Int., 81:02B111, 2010.

[4] Schiesko L., McNeely P., Fantz U. and Franzen P. and NNBI Team Plasma Phys. Control. Fusion, 53, 085029, 2011.

[5] Franzen P., Schiesko L., M. Fröschle, Wünderlich D., Fantz U. and NNBI Team Plasma Phys. Control. Fusion, 53, 115006, 2011.

[6] McNeely P and Wünderlich D. Plasma Sources Sci. Tech., 20, 045005, 2011.

[7] Franzen P. et al. Fusion Engeneering and Design, 82, 407, 2007.

[8] Fantz U. et al. AIP Conference Proceeding, 1097, 265, 2009.

[9] Tanga A., Bandyopadhyay M. and McNeely P. Appl. Phys. Lett., 84, 182, 2004.

[10] McNeely P., Dudin S. V., Christ-Koch S. and Fantz U. Plasma Sources Sci. Tech., 18, 014011, 2009.

[11] Lee B. S. and Seidl M. Appl. Phys. Lett., 61, 2857, 1992.

[12] Isenberg J. D., Kwon H. J., and Seidl M.. AIP Conference Proceeding, 287, 38, 1994.

[13] Simonin A. et al. Rev. Sci. Instrum, 67, 1102, 1996

[14] Fantz U. et al. Rev. of Sci. Instrum., 79, 02A511, 2008.

[15] Rust N. et al. Fus. Eng and Cont. Fusion (Montreux, Switzerland, 17-21 June 2002), published on CD-ROM.

[16] U. Fantz et al. Fusion Engeneering and Design, 74:399, 2005.

[17] Kiel R. AIAA J., 6, 708, 1968.

[18] Antonova A., Dudin S. V. and Farenik V. I. Problems of Atomic Science and Technology (Series: Plasma Physics vol 1) (Kharkov: Kharkov Institute of Physics and Technology),147-9.

[19] Chen F. F. Plasma Sources Sci. Tech., 18, 035012, 2009.

[20] Laframboise J. G. and Rubinstein J. Phys. Fluids, 19, 12, 1976.

[21] Schiesko L., Fantz U. and Franzen P. and NNBI Team Plasma Phys. Control. Fusion, To be published.

[22] Hanada M. et al Nucl. Fus., 46, S318, 2006.

[23] Boeuf J. P. et al. Plasma Sources Sci. Tech., 20, 015002, 2011.

[24] Hagelaar G. J. M et al. Plasma Sources Sci. Tech., 20, 015001, 2011.

[25] Kollev St, Lishev St., Shivarova A., Tarnev Kh. and Wilhelm R. Plasma Phys. Control. Fusion, 
Magnetic field dependance of the plasma properties in a negative hydrogen ion source for fusion 22 49, 1349, 2007.

[26] Stringer T. E. Plasma Phys. Cont. Fus., 33, 1175, 1991. 